\title{
Casa, hogar y objetos relacionais: resistencias al objeto como mercancía en la obra de Lygia Clark
}

\author{
House, home and objetos relacionais: resisting the \\ object as merchandise in the work of Lygia Clark \\ Moradia, lar e objetos relacionais: resistências ao \\ objeto como mercadoria na obra de Lygia Clark
}

\section{Sergio Delgado Moya}

HARVARD UNIVERSITY, ESTADOS UNIDOS

PhD en Spanish and Portuguese Languages and Cultures por Princeton University. Profesor asociado del Departamento de Español y

Portugués en Emory University. Entre sus principales publicaciones se encuentra Delirious Consumption: Aesthetics and Consumer Capitalism in Mexico and Brazil (University of Texas Press, 2017). Correo electrónico: sergiodelgadomoya@emory.edu

Artículo de reflexión

Este texto proviene del libro Delirious Consumption: Aesthetics and Consumer Capitalism in Mexico and Brazil (University of Texas Press, 2017).

Documento accesible en línea desde la siguiente dirección: http://revistas.javeriana.edu.co 


\section{Resumen}

Propongo ahondar en dos conceptos, hogar y objeto relacional, en tanto conciernen a la obra de Lygia Clark.

Comienzo con algunas observaciones sobre el interés de Clark en ideas propias de la arquitectura. Mi propósito es llegar a un entendimiento más complejo, más problemático y menos familiar de la casa, del hogar y sobre todo de los objetos que pueblan estos espacios; espacios clave para la consolidación de la cultura de consumo y para la construcción de nociones hoy en día operantes de lo que constituye la subjetividad.

Palabras clave: estética; arte; Brasil; neoconcretismo; consumo; hogar

\section{Abstract}

In this article, I would like to examine two concepts at play in the work of Lygia Clark: the concept of home and the notion of the "relational object". I begin with some observations about Clark's thoughts on architecture. My purpose is to arrive at a more complex, more problematic and less familiar understanding of the house, the home and, above all, of the objects that populate these spaces, which are key spaces for the consolidation of a culture of consumption and for the construction of current notions of what constitutes subjectivity. Keywords: aesthetics; art; Brazil; Neoconcretism; consumption; home

\section{Resumo}

Proponho aprofundar em dois conceitos, lar e objeto relacional, em tanto dizem respeito da obra de Lygia Clark. Começo com algumas observações sobre o interesse de Clark em ideias próprias da arquitetura. $\mathrm{O}$ meu propósito é chegar a uma compreensão mais complexa, mais problemática e menos familiar da moradia, do lar e sobre tudo dos objetos que habitam esses espaços, espaços-chave para a consolidação da cultura do consumo e para a construção de noções hoje em dia operantes do que constitui a subjetividade.

Palavras-chave: estética; arte; Brasil; neoconcretismo; consumo; lar

\section{Cómo citar este artículo:}

Delgado Moya, Sergio. "Casa, hogar y objetos relacionais: resistencias al

objeto como mercancía en la obra de Lygia Clark". Cuadernos de Literatura

22.43 (2018): 54-74. https://doi.org/10.11144/Javeriana.cl22-43.caho 
InTENTO ENTENDER LA obra de Lygia Clark y me viene a la mente una imagen: la artista en su casa, en su living, interactuando con sus "clientes" y con los objetos que Clark construía para dichos clientes en lo que fue la última etapa de su larga y prolífica trayectoria. Así la recuerda Yve-Alain Bois en su prefacio a una breve compilación de textos escritos por Clark (Clark y Bois). Así también la recuerda Suely Rolnik: "estamos en una sala en el apartamento de Lygia Clark en Rio de Janeiro. Un espacio inusual lleno de objetos de todo tipo, objetos que la artista llama 'relacionales" (Rolnik "Uma terapéutica"). ${ }^{1}$ La casa, el hogar como abrigo y como espacio de perturbación, es el punto de partida de la artista: Clark produce sus primeras obras en 1947, después de la crisis que le sobrevino tras el nacimiento de su tercer hijo. ${ }^{2}$ Tres décadas más tarde, pasadas largas estancias en París y después de un breve pasaje por California, Clark regresa a casa, a Rio de Janeiro, a la intimidad de su apartamento y a los ritmos dictados por esos espacios. En lo que resta de este ensayo, quiero proponer que la casa y el hogar, en el sentido más amplio de estos términos, son medidas aptas para la ambición de Clark, para su legado.

La preocupación por la forma y el concepto de la casa despunta en Clark desde comienzos de su trayectoria, desde sus primeras investigaciones sobre el encuadre y sobre el ambiente y hasta el desarrollo de los objetos relacionais, construidos por la artista con piedras, agua, plástico y otros materiales de uso doméstico. Es con base en esta preocupación que empiezan a emerger complicidades entre Clark y una serie de artistas (Sophie Taeuber, ${ }^{3}$ Liubov Popova, Alexander Rodchenko, Varvara Stepanova, Vladimir Tatlin) de la primera ola de vanguardias de comienzos de siglo veinte, complicidades fraguadas a partir de una común voluntad de romper con expectativas sobre el lugar de la obra de

$1 \quad$ Este dossier fue publicado como parte de la exhibición que lleva el mismo nombre y que abrió sus puertas en el Musée dês Beaux-Arts de Nantes en Francia en 2005 y en la Pinacoteca do Estado de São Paulo en 2006.

2 "Clark's artistic life began in 1947, in her own words, 'to survive the crisis' after the birth of her third child" (Rolnik, "Molding a Contemporary Soul" 67).

3 Citando a Hans Arp, compañero de Taeuber, Leah Dickerman define así el trabajo que ambos artistas llevaron a cabo durante los años de actividad de Dada en Zúrich: "Between 1916 and 1918, the couple developed a broad creative partnership, collaborating on paintings, collages, graphic work, and embroideries. Arp described their common goal as that of breaking with 'aesthetics' to produce work that would have more the status of ordinary objects. As part of this pursuit". Dickerman arguye, "Taeuber and Arp challenged the hierarchy between 'applied' and 'fine' art, often mobilizing the techniques and media of decorative arts in a deliberate shift toward a 'minor' key" (Dickerman 36). 
arte en el contexto cotidiano, y el lugar del trabajo doméstico -la decoración, las manualidades, el diario quehacer- en la producción de arte. Dado que fueron en su mayoría, y como en el caso de Clark, mujeres artistas las dedicadas de lleno a ese tipo de investigaciones, insistir sobre este gesto implica un riesgo: el riesgo de caer demasiado rápido en lugares comunes sobre el vínculo entre casa y mujer. Pasar por alto estas investigaciones implica quizás un riesgo mayor: el riesgo de obviar modos de revisión, de reconstrucción y reconceptualización de la casa y del hogar forjados justo al centro de la obra de algunas (y algunos) de las más consecuentes artistas de la vanguardia internacional.

Propongo pues ahondar en dichos modos en tanto conciernen a la obra de Clark. Comienzo con algunas observaciones sobre el interés de Clark en conceptos propios de la arquitectura (marcos, planos, líneas de encuadre, formas de abrigo, el espacio y la casa), para pasar después a una reflexión sobre el papel que el objeto y ciertas teorías sobre el objeto tuvo tanto para Clark como para muchos de sus contemporáneos neoconcretistas. ${ }^{4}$ Fue en referencia a la obra de Clark que el crítico y poeta Ferreira Gullar formuló en 196o su conocida teoría del no-objeto, cuyos presupuestos y repercusiones nos servirán para entender mejor la propuesta detrás de los objetos relaciones de Lygia Clark. De particular importancia para los propósitos de este ensayo es la doble labor desempeñada por muchos artistas concretos y neoconcretos contemporáneos a Clark, quienes participaron como artistas y como profesionales en industrias que hacia mediados del siglo XX comenzaban a consolidarse: la publicidad comercial, el diseño gráfico, el diseño industrial. Mi propósito es llegar a un entendimiento más complejo, más problemático y menos familiar de la casa, del hogar y sobre todo de los objetos que pueblan estos espacios; espacios clave para la consolidación de la cultura de consumo y para la construcción de nociones hoy en día operantes en la construcción de la subjetividad.

\section{Marcos, puertas, superficies penetrables}

El pensamiento arquitectónico figura de modo prominente en los primeros trabajos de Clark: dibujos y pinturas hechos bajo la tutela de Fernand Léger. En muchos de estos trabajos la imagen de la escalera y de su movimiento espiral aparecen como motivos principales. Según la misma Clark, sus retratos

4 El movimiento neoconcreto en Brasil surge en escena con la publicación del "Manifiesto neoconcreto" en el suplemento dominical de Jornal de Brasil, en marzo de 1959. Dicho manifiesto aparece firmado por Amilcar de Castro, Cláudio Mello e Souza, Ferreira Gullar, Franz Weissmann, Lygia Clark, Lygia Pape y Theon Spanudis. 
de escaleras y de otros motivos arquitectónicos aspiraban a ser recreaciones de espacios tangibles y materiales, espacios a ser habitados. "Comencé con la geometría [escribe Clark], pero lo que buscaba era un tipo de espacio orgánico que hiciera posible que alguien entrara en el mismo cuadro" (Wanderley 10). ${ }^{5}$ La arquitectura como forma y sensibilidad, como modo de estructurar el arte y como modo de pensar las vivencias que el arte hace posible fue la primera de una serie de disciplinas y discursos (la arquitectura y la industria en las décadas de $195^{\circ}$ y 1960; una forma rarificada de las manualidades en los años 60 y 70; la pedagogía y la psicología en los años 70 y 80) que fungieron como puntos de triangulación para Clark, puntos de fuga que le permitieron asumir una cierta distancia frente a las limitaciones discursivas, los métodos de producción y los mecanismos de recepción de la esfera del arte.

En 1947, en los albores de su carrera, Clark comienza un periodo de aprendizaje bajo la tutela del artista y arquitecto Roberto Burle Marx. Resulta poco sorprendente, pues, que la arquitectura y el pensamiento arquitectónico tengan una presencia tan fuerte en la obra temprana de Clark. Como señala Luis Pérez-Oramas, en sus primeros años de producción artística Clark parece aproximarse a la pintura como si fuera arquitectura, como si de una "ciencia topológica se tratara"; el sentido que Clark tenía de la pintura, Pérez-Oramas arguye, fue siempre "fundamentalmente topológico" (Pérez-Oramas 37). Algunos de sus trabajos exhiben este referente de modo manifiesto. Otros tienen una relación abstrusa pero inequívoca con la arquitectura, especialmente aquellas propuestas planteadas a partir de ropas o estructuras para ser usadas sobre el cuerpo, como abrigos. Hacia los años 60, Clark produce una serie de propuestas explícitas en su asimilación del lenguaje de la casa y del abrigo -Construa você mesmo seu espaço de viver (1960), Abrigo poético (1964), A casa do poeta (1967-1969), A casa é o corpo (1967-1969), Arquiteturas biológicas (1968-1969)-, propuestas que exploran metódicamente las preocupaciones que animan el interés de Clark por el discurso de la arquitectura: la idea del hogar, un sentido del quehacer entendido como poiesis, el cuerpo como abrigo y el abrigo -en tanto vestimenta y en tanto arquitectura- como cuerpo.

La búsqueda de Clark de un espacio pictórico pasible a ser penetrado o habitado redunda en una serie de maquetas arquitectónicas producidas en 1955 (Maquetes para interior), maquetas que recuerdan, en su austera

5 En el original: "Eu comecei com a geometria, mas buscava um espaço orgânico em que fosse possível para alguém entrar na pintura". 
y rítmica geometría al Proun Room que El Lissitzky construye en 1923. En Quebra da moldura (1954) Clark da un contundente paso hacia delante en esta búsqueda, sondeando la línea que divide lo que cabe y lo que queda fuera del plano pictórico, y explicitando desde el título de la obra el quiebre y la porosidad que Clark quiere producir a nivel visual y a nivel conceptual. Clark modula de manera precisa y metódica los bordes, los encuadres, la moldura y los límites de esta obra, activándolos de modo deliberado como elementos de la composición total. Cada borde, cada límite dentro y fuera del cuadro pide atención sin reclamarla, sin el desplante y la agresividad visual de, por ejemplo, las pinturas sobre molduras producidas en los años 40 en Buenos Aires por artistas asociados con el Grupo Madí y el movimiento Arte Concreto-Invención. El énfasis de Clark sobre los artefactos de encuadre y enmarque es más suave, más riguroso, más metódico y más disciplinado. El resultado es una superficie pictórica que se abre al espacio a su alrededor, gestionando hacia el muro donde cuelga la pintura y activando los quiebres, las aristas, los cortes y las hendiduras que marcan la arquitectura de dicho muro. La experiencia cultivada por esta quebra da moldura es notoriamente sensorial, marcada por una conciencia corpórea del espacio arquitectónico que nos rodea cuando nos encontramos adentro, en espacios interiores.

En un artículo de 1957, Oscar Niemeyer reconoce los vínculos que Clark busca establecer con el lenguaje y el pensamiento arquitectónico. Más aún, Niemeyer afirma que existe una dimensión social detrás del compromiso de Clark con la arquitectura. Según Niemeyer:

Lygia Clark es pintora de gusto y sensibilidad. No le bastan los problemas específicos de la pintura contemporánea. Su espíritu inquieto procura encontrar en los nuevos conceptos sociales un contenido nuevo para su trabajo. Busca una solución más ligada a los problemas constructivos, que permita una utilización más amplia y justa. Para Lygia Clark, los elementos arquitectónicos usuales -puertas, ventanas, etc.-, no constituyen obstáculos e interrupciones en la composición, sino que son módulos y ritmos capaces de enriquecerse o disciplinarse. (Clark, Ligia Clark 352)

La serie de pinturas acrílicas sobre madera de 1955, tituladas Superfícies moduladas, le dan seguimiento a la investigación arquitectónica en la obra de Clark. En estas pinturas, Clark abandona la tela y trabaja de lleno con paneles de madera enclavados, dejando atrás los materiales de producción propios de las bellas artes y adoptando métodos, materiales y herramientas 
de corte más industrial. ${ }^{6}$ El uso de planos de madera separados y enclavados dentro de un mismo cuadro abre divisiones reales y visibles entre cada bloque pictórico del cuadro, infundiendo en estas pinturas un sentido refrenado pero inquietante de profundidad, como si las divisiones, más que pictóricas, devinieran precipicios. El hecho de que las divisiones reales entre uno y otro panel de madera alternen con divisiones más llanamente pictóricas (bloques de colores dentro de un solo panel de madera) provoca un contraste y hace de las divisiones entre paneles una presencia aún más desconcertante.

Las Superficies moduladas de Clark cultivan en el espectador una postura distinta, un modo de atención diferente, contemplativo, pero ya tornado hacia la interacción y la participación, rasgos dominantes de la obra posterior de Clark. Verdes vivos, rojos ocre y azules cielo dominan la paleta de colores de esta fase en la pintura de Clark, un resabio, quizás, de los años formativos que la artista pasó bajo la tutela de Burle Marx. En apenas unos años, estos colores desaparecen de sus pinturas para dar paso a tonos más sombríos: negros, blancos y grises azulados. Estos colores son los que predominan en las series de pinturas rigurosamente geométricas que Clark produce en la segunda mitad de la década del 1950: Planos em superfície modulada, Espaços modulados y Unidades.

Al igual que las Superfícies moduladas, los Planos em superfície modula$d a$ están hechos con paneles de madera separados y enclavados. De cerca, se observan en la superficie delgadas aberturas con apariencia de fallas o fisuras, finas y rectas. Cada plano de la superficie pictórica está dispuesto de modo que sostiene la mirada ambivalentemente: el ojo retrocede y es absorbido por un mismo punto en la pintura. La experiencia visual aquí sostenida es ambigua, y un tanto inquietante. Cada vez que la mirada reposa sobre estas pinturas, surge una sensación de desazón muy parecida a la que provocan los ejercicios de ambivalencia perceptual citados con frecuencia en escritos sobre fenomenología y Gestalt (el conejo-pato de Wittgenstein, o el cubo de Necker). Los Planos em superfície modulada de Clark constituyen, en parte, investigaciones sobre este fenómeno, investigaciones contemporáneas a las que entonces llevaba a cabo Josef Alber con sus Structural Constellations. Tanto en las pinturas de Clark como en las de Alber (con quien Clark estudió durante una de sus estancias en París), la mirada del espectador es la vez

6 En una foto para un artículo de 1959 de la revista Querida, dirigida a un público femenino, Clark aparece con una pistola de pulverización de pintura en la mano: la viva imagen de la artista moderna, echando mano de los más recientes medios de producción. 
atraída y expulsada del plano pictórico en un juego perceptual que alterna entre impresiones de altura y protuberancia, por un lado, e impresiones de profundidad y concavidad, por el otro.

A través de esta alternancia, emerge una experiencia visual y perceptual más encopada, más propiamente corporal. Dependiendo de cómo uno ve cada superficie modulada, vemos los planos saliendo del plano pictórico o colapsándose a su interior -el mismo plano absorbe la mirada hacia el interior del cuadro, o bien la expulsa-. Emerge, pues, un sentido ambiguo del espacio pictórico como espacio a la vez envolvente y expulsivo, sentido del espacio que pronto se convierte en otro rasgo distintivo del trabajo de Clark, explicitado en obras como los Casulos de 1959 y 1960. Los Casulos son las últimas obras que Clark produce antes de construir los que habrían de convertirse en sus trabajos más conocidos: las esculturas plegables y sin base conocidas como Bichos (1960-1966). ${ }^{7}$ Desde un primer momento, los Bichos han sido celebrados por el modo en que ponen de manifiesto la mediación del cuerpo en la experiencia del arte y de la percepción, de la aisthesis en general. Pero ya en las pinturas geométricas de los años 50 se puede entrever tanto la importancia que Clark depositaba en la idea de la conciencia como pensamiento encarnado, como la agudeza con la que Clark logra apelar a este modo de la conciencia incluso en su obra más convencional.

La conferencia que Clark dictó en la Escola Nacional de Arquitetura de Belo Horizonte nos dice mucho sobre cómo la artista concebía la relación entre arte, cuerpo y arquitectura. Clark es oriunda de Belo Horizonte, y la ocasión de su conferencia marcó, de cierto modo, una vuelta a casa, a la ciudad natal, después de años viviendo en Rio de Janeiro. En su conferencia, Clark apuesta por el ambiente como el campo de operaciones compartido entre artistas e arquitectos. "Creo firmemente en la búsqueda de una fusión entre 'arte y vida'. No es posible que un hombre permanezca impasible ante el entorno en el que vive. Si el hombre busca la belleza y la armonía en una obra de arte individual, no existen razones para que no desee un ambiente armonioso para trabajar y vivir" (Clark, "Conferencia pronunciada" 71). La

"En octubre [de 1960], la artista presenta su serie Bichos en la Galería Bonino, con los siguientes títulos: Invertebrado, Ponta, Desfolhado, Articulado, Articulado duplo, Metamorfose I, Prisma, Metamorfose II, Vegetal, Constelação, Contrário I, Cidade, Contrário II, Vazado I, Vazado II, Sobre o redondo. La exposición obtiene un gran éxito y genera críticas numerosas y entusiastas en la prensa" (Clark, Lygia Clark 353). En esta obra puede encontrarse más información sobre la exhibición y recepción inicial de los Bichos. 
creciente porosidad entre arquitectura y arte coincide, según Clark, con la consolidación del arte concreto. Podríamos ir más lejos: la poesía concreta en Brasil parece ser el primer movimiento literario moderno en integrar de modo consistente y significativo el lenguaje de la arquitectura.

Para Clark, la ausencia de un "contenido expresivo" o el reconocimiento de la presencia de este tipo de contenido en aspectos de la obra de arte usualmente considerados como mero trasfondo marca la diferencia entre constructivismo y concretismo, por un lado, y otras formas de arte abstracto por el otro. Este reconocimiento es el punto de partida para Clark en su exploración del ambiente ${ }^{8}$ como más que mero plano de expresión, como algo que es "en sí mismo expresivo" (Pedrosa 72). El ambiente no como mero plano sino como sustancia de expresión: visión afín a reflexiones fenomenológicas (contemporáneas a las de Clark) sobre el cuerpo y sobre la relación entre figura y fondo. Filosóficamente, dicha visión libera el pensamiento de su yugo idealista, permitiéndole habitar de modo consecuente y productivo las dimensiones materiales y encarnadas de la experiencia (el lado "sucio" de la vida, según los criterios de Hegel). Esta misma visión es la que libera a Clark y a muchos de sus contemporáneos; sobre todo -aunque no exclusivamente- a artistas vinculados con el movimiento neoconcretista, que indagan de lleno en la dimensión de lo sensorial y la dimensión de lo material como expresiones plenas del pensamiento. Dichos artistas proceden con la certidumbre de que el trabajo en la sensación y en la materia no se agota en funciones preparativas o preliminares de la producción artística, como meros pasos hacia fines más consecuentes, hacia obra más acabada.

\section{Industria, empresa, producción en masa}

Una ola de optimismo y un sentido de posibilidad electrizante arrasa las mayores ciudades de Brasil en las dos décadas después de la Segunda Guerra Mundial, y ni Clark ni sus contemporáneos neoconcretistas estuvieron del todo exentos del anhelante espíritu de la época. ${ }^{9}$ Fueron muchas las

8 En un artículo de 1967, Mário Pedrosa postula la noción del ambiente, fundamental para artistas como Clark y Hélio Oiticica, como principio transversal de las ciencias, las artes y la tecnología (Pedrosa 135-138).

9 Las dos décadas después de la Segunda Guerra Mundial fue también el periodo dentro del cual se inauguran las grandes instituciones del arte brasileño: el Museu de Arte Moderna, MAM, en Rio de Janeiro (fundado en los años cuarenta y establecido de lleno en los cincuentas); el Museo de Arte de São Paulo, MASP (fundado en 1948); la Bienal de São Paulo (1951). Fue también durante estos años que la crítica de arte como tal se establece en Brasil, con el trabajo 
posibilidades, reales e ilusorias, que se abrieron para los artistas activos durante el gobierno de Kubitschek. Para los artistas, hubo sobre todo una apertura considerable hacia la industria como esfera de producción. Hay casos de artistas concretos trabajando en industria conocidos y bien estudiados, pero el cruce entre neoconcretismo e intereses comerciales es menos conocido y apenas debatido. Hasta hace relativamente poco, una buena parte de la crítica consideró apropiado distinguir de modo tajante entre el concretismo y el neoconcretismo, atribuyéndoles a los artistas y poetas concretos la sospechosa distinción de ser colaboradores en el mundo del comercio, a la vez que distanciaban a los artistas neoconcretos de todo contacto deliberado con dicho mundo. Pero este contraste, tan nítido, ha sido cuestionado recientemente por críticos atentos a los cruces y los puntos de contacto entre el concretismo y el neoconcretismo, por un lado, y entre ambos movimientos en su común apertura hacia la industria. Ronaldo Brito es uno de los críticos que defiende la división tajante entre artistas concretos y neoconcretos en términos de la relación que cada uno de estos grupos forjó, o no, con los intereses de la industria. Según Brito, el neoconcretismo "era prácticamente apolítico, se mantenía en un terreno reservado, era tímido y desconfiado en relación a la participación del arte en la producción industrial" (Brito 54). ${ }^{10}$

Muchos de los artistas y poetas que pasaron, parcial o permanentemente, a trabajar en industria en las décadas de 1950 y 1960 en Brasil estaban fuertemente vinculados al movimiento concreto, y permanecieron vinculados a él incluso después del corte que dio lugar a la creación del movimiento neoconcreto. Tal es el caso de Décio Pignatari y Waldemar Cordeiro. Otros (Abraham Palatnik, Aluísio Carvalho, Amilcar de Castro) pertenecen al grupo de artistas en Rio de Janeiro que rompieron con el movimiento concreto para pasar a formar parte del neoconcretismo. Otros como Mary Vieira y Almir Mavignier fueron de los primeros en suscribirse a la ola de abstraccionismo geométrico y concretismo que arrasó la escena de arte brasileña a comienzos

de figuras como Mário Pedrosa y Sérgio Milliet que formalizan la labor crítica en la esfera del arte, labor que hasta entonces se llevaba a cabo de manera más informal por poetas y personas allegadas a la literatura (ver Ferreira 14).

Brito formula sus criterios para distinguir y jerarquizar los movimientos concreto y neoconcreto en Brasil en un artículo de 1976, "Neoconcretismo". En 2006, en un volumen dedicado al concretismo en Brasil, el crítico brasileño lanzó un llamado a revisar la hipótesis de Brito, a someter dicha hipótesis a procesos de "verificación y profundidad" en vista de los visos esquemáticos que la lectura de Brito fue adquiriendo con el paso de los años (ver Mammì 26). 
de los años 50, ${ }^{11}$ para después partir hacia Europa. Todos ellos dejaron un corpus significativo de trabajo artístico más aptamente descrito como obra industrial o de diseño gráfico.

Son pocos los críticos y curadores que se han ocupado de esta parte de la producción concreta y neoconcreta. ${ }^{12} \mathrm{Y}$ hay razones contundentes para distanciar al neoconcretismo del mundo de la industria. En "Teoría do nãoobjeto" (1960) y en otros textos constituyentes del movimiento neoconcreto, impera lo que Sérgio B. Martins conceptualiza como un cierto historicismo teleológico (Martins 40), una voluntad de erigir el movimiento, de elevarlo, como realización máxima de la experimentación artística que surgió en Brasil desde las primeras décadas del siglo XX. De acuerdo a esta narrativa, la colusión entre intereses industriales y los valores estéticos del alto modernismo forjados en dicha experimentación es un paso en falso atribuible al arte y a la literatura concreta. El neoconcretismo, en cambio, se asume como una especie de reserva artística y estética frente a los avances del capitalismo cultural y de su lógica mercantilista.

Los Bichos de Clark, sus trabajos más conocidos y los más ampliamente discutidos entre la crítica, fueron concebidos en un primer momento como objetos de producción masiva y venta popular, en la calle y a través de vendedores ambulantes. Su destino fue otro, y hoy en día ocupan un lugar privilegiado en la historia del arte moderno: como ejemplos consagrados de un arte a la vez abstracto e interactivo, y como modelos de lo que Ferreira Gullar conceptualizó como el no-objeto. Los Bichos encarnan, como ninguna otra propuesta plástica de la época, las tres condiciones negativas que Mónica Amor define como características del no-objeto: no son pinturas, no son relieves y tampoco son esculturas (Amor 1). Gullar incluye otro rasgo en su definición del no-objeto: el no-objeto no es mercancía. Con todo, los Bichos de Clark, sus obras más conocidas, fueron concebidos en un primer momento como objetos de consumo, objetos para comprar y vender en la calle, indistinguibles en por lo menos un sentido (el sentido comercial) de lo que Gullar buscaba negar cuando acuña el concepto del no-objeto.

11 Según Ferreira Gullar, fue la Bienal de São Paulo (en particular, las delegaciones suizo-alemanas presentes en las primeras tres bienales) las que consolidaron el lenguaje de abstracción y la estética concreta en Brasil en los años cincuenta (ver Gullar, "Arte concreta no Brasil" 3).

12 Una excepción importante a esta tendencia: la exhibición de 2008 titulada Diálogo concreto, con curaduría de Daniela Name (ver Name). 
Este dato sobre las intenciones originales que Clark tuvo para sus $B i$ chos es poco conocido, a pesar de las enormes implicaciones que trae consigo el escenario hipotético de Bichos hechos para consumo masivo. En un diario inédito de 1963, Clark escribe:

mis planes iniciales para los Bichos no incluían museos ni marchants. Lo que yo quería era hacer muchos y venderlos hasta en las esquinas, a través de vendedores ambulantes. Mário Pedrosa consideró la idea un suicidio, pero bien que estoy arrepentida porque me parece que igual así debí haberlo hecho. $(\text { Clark, "1963-3") })^{13}$

Si Clark hubiera procedido con sus planes, algunos de los rasgos más intrigantes de estos objetos -su apelo a la participación, a un sentido de relatividad entre sujeto y objeto de arte; su sentido de juego libre- habrían quedado aún más enfatizados de lo que quedaron en la versión de los $B i$ chos que acabó siendo producida, la que terminó en museos y colecciones de arte. El riesgo, claro, habría sido trivializar los Bichos hasta el olvido. La producción en masa hubiera hecho de los Bichos un juguete curioso, lleno de intriga, bello en su construcción y consumadamente abstracto, una peculiaridad, otra rareza en la larga y distinguida trayectoria de una de las mayores artistas del siglo XX.

En relación a su propio interés y eventual adopción de métodos de producción industrial, Alexandre Wollner escribió: "Si dejé la pintura fue porque deseaba un diálogo no con diez, sino con mil o con un millón de personas, entre otras razones" (citado en Name 9). Entre artistas de la época, este deseo de una audiencia mayor llega a adquirir tonos llanamente políticos. En "Esquema geral da nova objetividade", el manifiesto escrito por Hélio Oiticica en respuesta a la exhibición titulada "Nova Objetividade Brasileira" en el Museu de Arte Moderna de Rio de Janeiro (1967), Oiticica define la "nueva objetividad" a partir de seis puntos principales: 1) una "voluntad general hacia lo constructivo"; 2) la "tendencia hacia la negación del objeto y la superación de la pintura al óleo"; 3) "participación del espectador (corpórea, táctil, visual, semántica, etc.)"; 4) "posicionamiento frente a problemas sociales, políticos y éticos"; 5) la "tendencia hacia propuestas colectivas", y

13 En el original: "Os meus planos iniciais para os bichos não incluíam Museus nem 'Marchands'. $\mathrm{O}$ que eu queria era fazer montes deles e pôr a venda até nas esquinas por camelô. Mário Pedrosa disse ser um suicídio mas bem que estou arrependida pois acho que era o que deveria ter feito mesmo". 
6) la "reemergencia y nueva formulación del concepto de anti-arte" (Oiticica). La producción de obras de arte abiertas, ${ }^{14}$ interactivas (a eso se refiere Oiticica, como Mário Pedrosa y Ferreira Gullar, con el término "anti-arte") está al centro de lo que Oiticica llama "nueva objetividad", como también lo está la necesidad de aumentar exponencialmente el orden de producción y de consumo de la experiencia artística. El artista, arguye Oiticica:

siente una necesidad mayor, no sólo de criar simplemente, sino de comunicar algo que para él es fundamental, pero esa comunicación tendría que darse en gran escala, no para una élite reducida a "expertos" sino más bien en contra de esa élite, con la propuesta de obras no acabadas, "abiertas". Ese es un aspecto fundamental del nuevo concepto de anti-arte: no solamente insistir contra el arte del pasado o contra los conceptos antiguos [...] sino más bien crear nuevas condiciones experimentales, en las que el artista asuma el papel de "propositor", o "empresario" o incluso "educador". (Oiticica 17)

Para entonces, la noción del artista como propositor ya estaba al centro de la estética neoconcretista y había sido adoptada y teorizada con particular vigor por Oiticica y por Clark. La idea del artista como educador, por otro lado, evoca otros linajes de vanguardia, visiones como la de László Moholy-Nagy, profesor de la Bauhaus, que definió el arte como "educación de los sentidos". Lo que realmente sorprende es la franqueza con la que Oiticica conjuga el papel del artista con el rol del empresario. Para él como para Wollner y para Clark, la necesidad de un diálogo más amplio, de un diálogo masivo y más horizontal de lo que permiten los métodos de producción y circulación convencionales del arte, es mayor que cualquier resabio que pueda causar la colusión de la estética y de la industria, el colapso entre el arte en su calidad de expresión no-determinada y la industria como la producción de lo práctico y lo utilitario (o peor aún: como la producción de lo comercial). Oiticica nunca clarificó del todo qué exactamente implica la conjugación de los papeles del artista y el empresario, pero el hecho de que haya considerado esta conjugación como posibilidad nos dice mucho de las insinuaciones existentes entre el neoconcretismo, la industria y la cultura de masas, insinuaciones mucho menos fáciles de entender y clasificar de lo que supone mucha de la crítica que hasta ahora se ha dedicado a narrar la historia del neoconcretismo.

14 Haroldo de Campos elabora el concepto de la obra de arte abierta en Brasil en 1955, siete años antes de la publicación del libro de Umberto Eco sobre el mismo tema (Campos 30-33). 


\section{Objetos relacionales, "gestos cotidianos" y el fetichismo de la mercancía} No es inusual encontrar, en la crítica sobre la obra de Lygia Clark, un cierto asombro ante la entereza con la cual la artista procede de una etapa de su investigación a otra. Se dibuja un desarrollo claro y contundente en su progresiva preocupación con la interactividad, con la participación, con la corporalidad y con la abstracción como elementos fundamentales de la experiencia artística. La última etapa de su investigación es más difusa, mucho menos maleable. Comienza con la propuesta titulada Caminhando (1963), el primer intento de Clark por desalojar sus propuestas del objeto como soporte, como forma estable, su primera incursión en el tipo de experiencias rituales, meditativas y de inmersión que vendrían a caracterizar los últimos veinticinco años de su producción artística. Clark le dio un nombre a la etapa de su trayectoria en la cual produjo Caminhando:

Esta fase de mi trabajo, la más variada de todas, la he llamado: Nostalgia do corpo. Después he comprendido que una de las propiedades del Caminhando estaba aquí radicalizada: la proposición (porque la palabra "obra", al denotar la pasividad del resultado de un trabajo anterior, no conviene aquí) nos hace tomar conciencia de nuestro propio cuerpo. Reuní un gran número de materiales sin valor cuya aprehensión, el redescubrimiento táctil, provocaba un trauma estimulante. (Clark, "Encontrar el sentido" 188)

Después de Nostalgia do corpo, el redescubrimiento de aquello que pasamos por alto (el trasfondo material, cotidiano, de poco valor, de la vida diaria) se convierte en uno de los principales motores de la obra de Clark. El propósito de este redescubrimiento no se agota en dicha materialidad. El apelo a una conciencia encarnada y a una mayor consciencia del cuerpo como matriz de la consciencia son los principales propósitos detrás de la serie de objetos "relacionales" que Clark comienza a construir a partir de 1966. Todas las propuestas que Clark formuló en las últimas dos décadas de su producción están estructuradas alrededor de estos objetos de poco valor. La insistencia con la que regresa una y otra vez a estos objetos exige una lectura atenta.

Una vez que Clark comienza a trabajar de lleno en la producción de sus objetos relacionales, la labor que dicha producción requiere arrima el trabajo de Clark -extrañamente, como si de un experimento o de un laboratorio se tratara- al mundo de las manualidades, al tipo de labor que asociamos con el espacio doméstico. Ya en la producción de los Bichos y en la última fase de su pintura, Clark se había distanciado de los modos de producción artesanales 
propios de las bellas artes. Con los objetos relacionales, Clark se ciñe a esta dirección, distanciándose más y más de los métodos convencionales de la producción artística y acercándose cada vez a una forma rarificada y experimental del trabajo manual con materiales desechables.

Clark comienza a trabajar en su nueva visión del objeto, el objeto relacional, en París en los años sesenta, bajo condiciones de marcada precariedad. "A través de pequeños objetos sin valor como gomas elásticas, piedras, bolsas de plástico [escribe Clark], formulo objetos sensoriales cuyo tocamiento provoca sensaciones que identifico inmediatamente con el cuerpo" (Clark, "De la supresión del objeto" 266). Clark narra el comienzo de esta fase de su trabajo en una carta dirigida a Hélio Oiticica. "Ya empecé a trabajar catando piedras en las calles, iy es que no hay dinero para comprar material! Uso todo lo que cae en mis manos, sacos vacíos de papas, de cebollas, los plásticos que cubren la ropa de la tintorería, iy hasta los guantes de plástico que uso para pintarme el cabello!" (Clark y Oiticica 36 ). ${ }^{15}$ Articula el andamiaje teórico de sus objetos relacionales en retrospectiva, en un artículo de 1974 titulado "Da supressão do objeto (anotações)" publicado en el legendario y único número de la revista Navilouca. El título del artículo nos da una medida de las contradicciones que barajaba Clark en su concepción del objeto. Según Clark, el objeto había perdido "su capacidad como plataforma de comunicación", pero, aun así, Clark atribuía al objeto una capacidad superior de vinculación.

$\mathrm{Al}$ mismo tiempo que construía sus objetos relacionales, Clark estaba cada vez más consciente del potencial político de su trabajo. Para Clark, el impacto inmediato que los objetos relacionales podían tener en los eventos sociales y políticos de la época yace en su capacidad de dirigir nuestra atención y conciencia hacia "gestos cotidianos". Escribe Clark:

Es durante esta época, cuando el carácter político y social de mi trabajo se hace evidente para mí: ya que él se realizaba a partir de una liberación del hombre, del levantamiento de una represión, puesto que el participante encontraba una energía sensorial voluntariamente adormecida por nuestros hábitos sociales, estas experiencias tenían un impacto revolucionario y, por otra parte, eran recibidas como tales. (Clark, "Encontrar el sentido" $188-189$ )

15 En el original: "Comecei já a trabalhar catando pedras nas ruas, pois dinheiro não há para comprar material! Uso todo que me cai nas mãos, como sacos vazias de batatas, cebolas, plásticos que envolvem roupas que vêm do tintureiro, e ainda luvas de plástico que uso para pintar os cabelos!"”. 
Gran parte del potencial político de los objetos relacionales de Clark recae también en el modo en que resisten, o más bien en el modo en que le sacan la vuelta, al lenguaje verbal. No es que los objetos relacionales escapen o funcionen totalmente fuera del lenguaje, del orden simbólico. Clark era demasiado astuta como para comprometerse con la posibilidad de una posición concebida fuera del orden del lenguaje. El gesto más bien consiste en abrir la puerta a un modo de liberación (de energías, de conciencia, de deseos, de posibilidades) que no pase en primera instancia por el lenguaje, y que no sea tampoco un medio preparatorio hacia una articulación hecha plena solo después de su verbalización. La verbalización, el retorno al lenguaje, funge como un paso importante en el ejercicio clínico que Clark llegó a plantear en torno a sus objetos relacionales, la Estruturação do self, con la que se ocupó en la última década de su trayectoria. Pero la idea principal, y en esto Glark fue tajante, es abrir posibilidades de expresión y percepción menos marcadamente jerarquizadas por el orden simbólico, posibilidades rigurosas pero menos disciplinadas y potencialmente mucho más desestabilizantes, en tanto intensivas.

Hasta hace poco, mucha (si bien no toda) la crítica sobre la obra de Clark se mostró un tanto tímida ante la dimensión política del trabajo de la artista brasileña. Pero existe una ola reciente de investigaciones que insisten en resaltar el sentido político latente y en ocasiones explícito en la obra de Clark y en el movimiento neoconcreto en general. Como apunta Sérgio B. Martins, Mário Pedrosa, el principal teórico del movimiento neoconcreto, "fue militante trotskista... comprometido con la transformación política" y "convencido del rol que el arte juega en este proceso de transformación no es simplemente un rol ilustrativo" (Martins 23). En el caso de Clark, la presencia de una dimensión política más o menos visible, más o menos articulada, responde a las categorías que utilizamos para distinguir lo político de lo "apolítico" en el arte, y no a algún rasgo inherente a la obra de Clark en particular o a la obra de arte en general. En el caso de Clark, lo urgente pues no es tanto marcar la falta o la presencia de una dimensión política; lo que urge es encontrar un lenguaje que nos permita determinar esta falta o esta presencia de modo más informado. En años recientes, los lenguajes que nos pueden ayudar con esta tarea se vienen acumulando: el lenguaje de la micro-política como lo teoriza Suely Rolnik en referencia al trabajo de Gilles Deleuze y Félix Guattari; el lenguaje de la política de la sensación que imagina Davide Panagia, la política como dimensión de lo sensorio (ver Panagia) -como plano de intervención sensorial en eventos 
sociales de actualidad-; y el lenguaje revisado de la casa, del hogar y de los objetos que pueblan dicho espacio que planteo yo en el presente ensayo. Todos estos lenguajes generan, en diferentes medidas y a diferentes escalas, lecturas politizadas de la obra de Clark.

He aquí otro modo de concebir la valía política de la obra de Clark y de sus objetos relacionales en particular, basado en el redescubrimiento de la "energía sensorial" latente en los gestos cotidianos. A través de la obra de Clark, y en particular gracias al tipo de atención que sus propuestas cultivan, nuestro modo de percibir y de estar conscientes de los objetos, los sujetos y las relaciones entre sujetos y objetos que pueblan nuestra vida cotidiana se transforma de modo consecuente, haciendo que toda una dimensión (la dimensión material y sensorial, el trasfondo) de nuestros gestos cotidianos y de los objetos y sujetos involucrados en dichos gestos pase a primer plano. El término "objetos relacionales" ya indica lo más importante de estos objetos: no el objeto en sí (precario, efímero, sorprendente en su simplicidad material y en la fragilidad de su construcción, desnudado de cualquier trazo que le pueda infundir permanencia o valor material como objeto de arte), sino más bien las relaciones que dicho objeto puede mediar. Los objetos relacionales operan a través de la sensación. Operan al nivel de lo sensible. Permanecen impasibles a no ser que sean manipulados, lo cual no quiere decir que sean inertes o del todo pasivos.

La importancia del trabajo de Clark con los objetos relacionales y las posibles implicaciones políticas de dicho trabajo son más nítidas en referencia a lo que Michael Taussig denomina "el dominio de la cosidad" -"the dominance of thinghood" (Taussig 35)-, una ontología y también una estructura de poder perpetuadas cuando los principios de identidad y de auto-contención son defendidos por sobre las relaciones sociales y humanas. Para Taussig, la "dominancia de la cosidad" toma lugar cuando las condiciones de labor y producción quedan subyugadas a la lógica del capitalismo. La cosidad como ontología gana más terreno con la diseminación de los productos del sistema de producción capitalista, productos investidos con un nivel de abstracción y autocontención tan altos como el tiempo-labor requerido para su producción. La teoría de los objetos y la idea de objetividad que emerge del movimiento neoconcreto en general y de la noción de los objetos relacionales en particular puede ser leída en oposición y contraste a este tipo de diseminación.

Un análisis detenido de las figuras y las estructuras de la sociedad de consumo, trasfondo general de la producción de Clark, revela la necesidad 
de revisar la antinomia objeto-sujeto, de modo que ciertas imbricaciones, confusiones y hasta transposiciones de las figuras del objeto y del sujeto puedan ser reconocidas como algo más que el resultado de una consciencia alienada, algo que quizás es lo contrario de este modo de conciencia: algo así como una liberación, en la más polémica de las posibles lecturas. A través de la objetivización consciente, del devenir objeto (distinto, en este caso, de la alienación como condición de subjetividad), podemos empezar a dibujar los contornos de una ética concreta: el reconocimiento y el cuidado de algo más que el sí mismo, algo que es, en primera instancia, otro objeto, otro cuerpo, otra materia. La consideración de la textura y de otras experiencias sensoriales intensivas sería la base de esta nueva ética, del todo estética. Argüir, pues, por una reivindicación de la sensación y lo sensorial, a la vez que reconocemos la sensación y lo sensorial como modos primarios de apelación en el capitalismo de consumo, no significa retomar relaciones cómplices o conformes con la cultura de consumo. Más bien, significa librar al objeto del yugo que le ha impuesto su estatus como mercancía, el yugo que dicho estatus le ha impuesto, por extensión, a nuestras relaciones con los objetos. Esto, claro está, debe ser concebido en términos que no se agoten en nuestra relación con los objetos, en términos que abarquen también las relaciones que establecemos entre nosotros mismos a través de los objetos que producimos, que intercambiamos y que consumimos.

Vistos de tal modo, los objetos relacionales de Clark nos llevan a repensar la dimensión relacional de nuestra experiencia en el mundo y nuestra interacción con otros sujetos y otros objetos. Funcionan como aquello que Irene V. Small concibe, en referencia a la obra de Oiticica, como "artefactos epistemológicos, es decir, modelos materiales y encarnados de conocimientos emergentes" (Small 7). ${ }^{16}$ Como artefactos epistemológicos, los objetos relacionales revelan nuevas formas del conocimiento y modelan formas del sentir, formas desafiantes por el modo en que cultivan la materialidad justo en el momento en que la cultura material comienza a desplegarse implacablemente para fines utilitarios y comerciales. La cultura de consumo prospera ante la creciente presencia de objetos y la labor de Clark con sus objetos relacionales está plenamente consciente de esta floreciente diseminación de los objetos. El énfasis en la teoría neoconcreta sobre la naturaleza relacional del no-objeto, un énfasis puesto de manifiesto en los objetos relacionales

16 En el original: "an epistemological device, that is, as a material, embodied model of emergent knowledge". 
de Clark, bien puede ser leído como un gesto de resistencia, o mejor aún, como un enjuiciamiento de la supuesta autocontención del objeto como mercancía, la aparente naturalidad de su separación, de su abstracción, de los contextos de producción y consumo que dan lugar a su existencia. Los objetos relacionales de Clark también pueden ser leídos como formas de contestación frente a la noción de subjetividad que corresponde a esta ontología de objetos como entidades autocontenidas y ensimismadas. Vistos así, los objetos relacionales de Clark emergen como formas de resistencia a lo que Rolnik describe como "la figura del 'individuo', una entidad auto-contenida que deriva su sensibilidad de una imagen vivida como esencia, imagen mantenida en estricta identificación con sí misma, inmune a la alteridad y sus turbulentos efectos" (Rolnik, "Molding a Contemporary Soul" 63). Los objetos relacionales y la teorización de la noción de objetos llevada a cabo por Gullar, por Pedrosa, por Oiticica y por Clark funcionarían, pues, como modos de imaginar la naturaleza relacional no solo de la experiencia, sino de la misma constitución tanto del sujeto como de los objetos que pueblan y median la experiencia subjetiva.

\section{Obras citadas}

Amor, Mónica. Theories of the Nonobject: Argentina, Brazil, Venezuela, 19441969. Oakland, California: University of California Press, 2016. Impreso.

Brito, Ronaldo. "Neoconcretismo". Malasartes 3 (abril-junio 1976): 9-13. Impreso.

Brito, Ronaldo. Neoconcretismo: vértice e ruptura do projeto construtivo brasileiro. São Paulo: Cosac \& Naify Edições, 1999. Impreso.

Campos, Haroldo de. "A obra de arte aberta". 1955. Teoria da poesia concreta: textos críticos e manifestos 1950-1960. São Paulo: Ateliê Editorial, 2006. 30-33. Impreso.

Clark, Lygia. "1963-3". Sin fecha. Archivos del Clark Art Center, Rio de Janeiro, Brasil.

Clark, Lygia. "Conferencia pronunciada en la Escuela Nacional de Arquitectura de Belo Horizonte en 1956". Lygia Clark. Barcelona: Fundació Antoni Tápies, 1998. 71-73. Impreso.

Clark, Lygia. "De la supresión del objeto (apuntes)". Lygia Clark. Barcelona: Fundació Antoni Tápies, 1998. 264-269. Impreso.

Clark, Lygia. "Encontrar el sentido de nuestros gestos rutinarios". Lygia Clark. Barcelona: Fundació Antoni Tápies, 1998. 188-189. Impreso.

Clark, Lygia. "Eu, Lygia Clark". N.d. TS. Archives of the Clark Art Center, Rio de Janeiro, Brazil. Impreso.

Clark, Lygia. Lygia Clark. Barcelona: Fundació Tàpies, 1998. Impreso. 
Clark, Lygia. "O objeto, o método e a prática". N.d. TS. Archives of the Clark Art Center, Rio de Janeiro, Brazil. Impreso.

Clark, Lygia e Yve-Alain Bois. "Nostalgia of the Body". October 69 (Summer, 1994): 85-109. Impreso.

Clark, Lygia y Hélio Oiticica. Cartas: 1964-1974. Ed. Luciano Figueiredo. Rio de Janeiro: Editora UFRJ, 1996. Impreso.

Dickerman, Leah. "Zurich". Dada: Zurich, Berlin, Hannover, Cologne, New York, Paris. Leah Dickerman. Washington D.C.: National Gallery of Art, 2005. 16-44. Impreso.

Ferreira, Glória. "The Permanent Revolution of the Critic". Mário Pedrosa: Primary Documents. Eds. Glória Ferreira y Paulo Herkenhoff. Nueva York: Museum of Modern Art, 2015. 14-23. Impreso.

Gonzalez, Mike and David Treece. The Gathering of the Voices: The TwentiethCentury Poetry of Latin America. Verso: London: 1992. Impreso.

Gullar, Ferreira. "Arte concreta no Brasil: etapas da pintura contemporânea XXXIX - arte concreta V". Fornal do Brasil: suplemento dominical (6 de agosto de 1960): 3. Impreso.

Gullar, Ferreira. "Teoria do não-objeto". 196o. Projeto construtivo brasileiro na arte: 1950-1962. Ed. Aracy Amaral. São Paulo: Pinacoteca do Estado de São Paulo, 1977. 85-94. Impreso.

Mammì. "Concrete '56: The Root of Form (A Reconstruction of the I National Exhibition of Concrete Art)". Concreta '56: a raiz da forma. Eds. Lorenzo Mammì et al. São Paulo: Museu de Arte Moderna de São Paulo, 2006. 22-50. Impreso.

Martins, Sérgio B. Constructing an Avant-Garde: Art in Brazil, 1949-1979. Cambridge, MA: MIT Press, 2013. Impreso.

Müller, Ulrike. Bauhaus Women: Art, Handicraft, Design. London: Thames \& Hudson, 2009. Impreso.

Name, Daniela. Diálogo concreto: design e construtivismo no Brasil. Rio de Janeiro: Caixa Cultural, 2008. Impreso.

Oiticica, "Esquema geral de nova objetividade". NOVA objetividade brasileira. Rio de Janeiro: Museu de Arte Moderna do Rio de Janeiro, 1967. 4-18. Impreso.

Panagia, Davide. The Political Life of Sensation. Durham, NC: Duke University Press, 2009. Impreso.

Pedrosa, Mário. 1967. "World in Crisis, Man in Crisis, Art in Crisis". Primary Documents. Eds. Glória Ferreira y Paulo Herkenhoff. Nueva York: Museum of Modern Art, 2015. 135-138. Impreso.

Pérez-Oramas. "Lygia Clark: If You Hold a Stone". Lygia Clark: The Abandonment of art, 1948-1988. Nueva York: Museum of Modern Art, 2014. Impreso. 
Rolnik, Suely. "Molding a Contemporary Soul: The Empty-Full of Lygia Clark". The Experimental Exercise of Freedom: Lygia Clark, Gego, Mathias Goeritz, Hélio Oiticica, Mira Schendel, organizado por Rina Carvajal y Ana Ruiz, 59-98. Los Angeles: Museum of Contemporary Art, 1999. Impreso.

Rolnik, Suely. "Uma terapêutica para tempos desprovistos de poesia". Lygia Clark da obra ao acontecimento: somos o molde. A você cabe o sopro. São Paulo: Pinacoteca do Estado de São Paulo, 2006. Impreso.

Small, Irene V. Hélio Oiticica: Folding the Frame. Chicago: University of Chicago Press, 2016. Impreso.

Taussig, Michael T. The Devil and Commodity Fetishism in South America. Chapel Hill: University of North Carolina Press, 1980. Impreso.

Wanderley, Lula. O dragão pousou no espaço: arte contemporânea, sofrimento psíquico e o Objeto Relacional de Lygia Clark. Rio de Janeiro: Rocco, 2002. Impreso. 